\title{
MiR-144 Inhibits Tumor Growth and Metastasis in Osteosarcoma via Dual-suppressing RhoA/ROCK1 Signaling Pathway
}

\author{
Jin Long Liu, Jing Li, Jia Jia Xu, Fei Xiao, Peng Lei Cui, Zhi Guang Qiao, Xiao Dong Chen, \\ Wei Dong Tao, and Xiao Ling Zhang
}

Department of Orthopedic Surgery, Xin Hua Hospital Affiliated to Shanghai Jiao Tong University School of Medicine (SJTUSM) (J.L.L., J.J.X., F.X., P.L.C., X.D.C., W.D.T., X.L.Z.); Key Laboratory of Stem Cell Biology, Institute of Health Sciences, Shanghai Institutes for Biological Sciences, Chinese Academy of Sciences \& Shanghai Jiao Tong University School of Medicine (J.L.); Surgical Department, Kunshan Traditional Medicine Hospital (W.D.T.); and Shanghai Key Laboratory of Orthopedic Implant, Department of Orthopaedic Surgery, Shanghai Ninth People's Hospital, Shanghai Jiao Tong University School of Medicine (Z.G. Q.), Shanghai, People's Republic of China

Received September 11, 2018; accepted January 12, 2019

\section{ABSTRACT}

Several microRNAs (miRNAs) have been found expressed differentially in osteosarcoma (OS), so they may function in the onset and progression of OS. In this study, we found that miR144 significantly suppresses osteosarcoma cell proliferation, migration, and invasion ability in vitro and inhibited tumor growth and metastasis in vivo. Mechanically, we demonstrated that Ras homolog family member A (RhoA) and its pivotal downstream effector Rho-associated, coiled-coil-containing protein kinase 1 (ROCK1) were direct targets of miR-144. Moreover, the negative correlation between down-regulated miR-144 and up-regulated ROCK1/RhoA was verified in both OS cell lines and clinical patients' specimens. Functionally, RhoA with or without ROCK1 co-overexpression resulted a rescue phenotype on miR-144 inhibited cell growth, migration, and invasion abilities whereas individual overexpression of ROCK1 had no statistical significance compared with controls in miR-144-transfected SAOS2 and U2-OS cells. Taken together, this study demonstrates that miR-144 inhibited tumor growth and metastasis in OS via dual-suppressing of RhoA and ROCK1, which could be a new therapeutic approach for the treatment of OS.

\section{Introduction}

Osteosarcoma (OS) is the most prevalent bone tumor in children and young adults (Bielack et al., 2008). Most tumors arise from the metaphysis of the long bones and easily metastasize to the lungs (Harting and Blakely, 2006). The current therapeutic strategies of OS are routinely surgical resection and chemotherapy (Link et al., 1986), which are limited to the patients with metastatic recurrence. Survival of OS patients remains poor, and the 5-year survival rate for patients who have metastasis is about 15\%-30\% (Mirabello et al., 2009). Investigating the molecular mechanisms that contribute to OS progression is important and may shed light on a targeted therapeutic approach to improve the survival of patients with this disease.

This work was supported by grants from National Natural Science Foundation of China (No. 81830078, 81772347, 81572123), Science and Technology Commission of Shanghai Municipality (No. 16430723500), Shanghai Municipal Education Commission-Gaofeng Clinical Medicine Grant Support (No. 20161314) and Shanghai Shen Kang hospital development center (No. 16CR2036B).

https://doi.org/10.1124/mol.118.114207.
MicroRNAs (miRNAs) are small noncoding single-stranded RNAs that repress gene expression post-transcriptionally by binding to the $3^{\prime}$-untranslated region $\left(3^{\prime}\right.$-UTR) of their target mRNAs (Bartel, 2004). MiRNAs play critical roles in physiologic processes such as cell growth, development, and differentiation (Alvarez-Garcia and Miska, 2005). Also, aberrant expression of specific miRNAs leads to the onset and/or deterioration of many diseases, such as cancer (Croce, 2009), heart disease (Divakaran and Mann, 2008), and arthritis (Tili et al., 2008). Recently, several miRNAs have been identified as exerting anticancer effects by influencing cell proliferation, apoptosis, and migration, acting as oncogenes or tumor suppressors (Lu et al., 2005; Hurst et al., 2009). For example, miR-181a has been reported to be up-regulated in OS and to correlate with cancer development (Jianwei et al., 2013).

Rho-associated, coiled-coil containing protein kinase (ROCK) is a key downstream effector of the small GTPase Ras homolog family member A (RhoA). Two ROCK isoforms, ROCK1 and ROCK2, have been identified in mammalian cells and share $65 \%$ identity in their amino-acid sequences (Nakagawa et al., 1996). ROCK1 has a ubiquitous distribution, and ROCK2 is mainly expressed in the brain and muscle tissue (Hahmann

ABBREVIATIONS: 3'-UTR, 3'-untranslated region; $\alpha$-MEM, minimum essential medium $\alpha$; miRNAs, microRNAs; CCK-8, Cell Counting Kit-8; FACS, fluorescence-activated cell sorter; jet-PEI, jet-polyethylenimine; LIMK, LIM kinase; miR-NC, negative control miRNA; OS, osteosarcoma; $\mathrm{PCR}$, polymerase chain reaction; qRT-PCR, quantitative reverse-transcription polymerase chain reaction; RhoA, Ras homolog family member $\mathrm{A}$; ROCK1, Rho-associated, coiled-coil containing protein kinase 1; 3'-UTR, 3'-untranslated region; Y-27632, 4-[(1 \{R\})-1-aminoethyl]- $\{N\}$-pyridin-4ylcyclohexane-1-carboxamide. 
and Schroeter, 2010). When activated by Rho-GTP, ROCK phosphorylates several substrates, such as myosin light chain 2 (Leung et al., 1996), LIM kinase 1 (LIMK) (Ohashi et al., 2000), and LIM kinase 2 (LIMK2), which leads to the phosphorylation of cofilin (Maekawa et al., 1999). The RhoA/ROCK pathway mediates a number of cellular functions, including cytoskeleton reorganization and cell mobility, proliferation, and survival. Elevated ROCK1 expression has been detected in a spectrum of malignancies, such as breast (Cimino et al., 2013), prostate (Lin et al., 2008), and gastric (Zheng et al., 2011) cancers, and this deregulation usually correlates with a poor prognosis.

Previous studies have shown that ROCK1 is strongly up-regulated in OS clinical specimens (Liu et al., 2011), which makes it a potential therapeutic target with this disease. We wondered whether the deregulation of ROCK1 was associated with miRNA regulation. In this study, we identified miR-144 as a negative regulator of ROCK1 and RhoA by in silico miRNA target prediction algorithms. We demonstrated that miR-144 was down-regulated in OS clinical specimens and cancer cell lines. We revealed for the first time that miR-144 repressed tumorigenesis and metastasis through dualsuppression of RhoA/ROCK1 pathway. Our results indicated that miR-144 plays a critical tumor-suppressive role in the process of OS progression and could be a potential candidate for the treatment of OS.

\section{Materials and Methods}

Tissue Specimens. Tissue samples from 51 OS patients were obtained from Shanghai Ninth People's Hospital. The details of the cases are presented in Table 1 . As a control, normal human bone fragments isolated from healthy donors who had undergone total knee arthroplasty were obtained from Shanghai Ninth People's Hospital. Informed consent was obtained from all patients before conducting the study. The tissues were fixed with $4 \%$ paraformaldehyde for histopathologic diagnosis and immunohistochemical staining.

Cell Culture. All cell lines were obtained from the American Type Culture Collection (ATCC, Manassas, VA). Human osteoblastic cell

TABLE 1

Association between miR-144-3p expression and characteristics of patients with osteosarcoma

$P$ values were obtained from Pearson's chi-squared test or Fisher's exact test.

\begin{tabular}{|c|c|c|c|c|}
\hline \multirow{2}{*}{ Variables } & \multirow{2}{*}{ No. of Cases } & \multicolumn{2}{|c|}{ Expression of miR-144-3p } & \multirow{2}{*}{$P$ Value } \\
\hline & & Low & High & \\
\hline Age & & & & 0.504 \\
\hline$<21$ & 25 & 12 & 13 & \\
\hline$\geq 21$ & 26 & 11 & 14 & \\
\hline \multicolumn{5}{|l|}{ Gender } \\
\hline Male & 33 & 18 & 15 & 0.639 \\
\hline Female & 18 & 10 & 8 & \\
\hline Location & & & & 0.412 \\
\hline Distal & 16 & 9 & 7 & \\
\hline Proximal & 35 & 19 & 16 & \\
\hline Histologic type & & & & \\
\hline Osteoblastic & 41 & 18 & 23 & \\
\hline Chondroblastic & 5 & 2 & 3 & \\
\hline Fibroblastic & 5 & 3 & 2 & \\
\hline TNM stage & & & & $<0.001$ \\
\hline $\mathrm{I}+\mathrm{II}$ & 31 & 10 & 21 & \\
\hline III+IV & 16 & 11 & 5 & \\
\hline Distant metastasis & & & & $<0.001$ \\
\hline No & 22 & 14 & 8 & \\
\hline Yes & 29 & 23 & 6 & \\
\hline
\end{tabular}

line hFOB 1.19 was maintained at $33.5^{\circ} \mathrm{C}$ in Dulbecco's modified Eagle's medium/Ham's F-12 medium (GIBCO, Grand Island, NY) supplemented with 10\% FBS (GIBCO). Human OS cell lines SaOS2, $\mathrm{U} 2 \mathrm{OS}$, and MG63 were cultured in minimum essential medium $\alpha$ $\left(\alpha\right.$-MEM; GIBCO) supplemented with $10 \% \mathrm{FBS}(\mathrm{GIBCO})$ at $37^{\circ} \mathrm{C}$ in humidified atmosphere containing $5 \% \mathrm{CO}_{2}$.

Cell Viability Assay. Cell growth was monitored with the CCK-8 assay as per the manufacturer's instructions. Briefly, cells at $5 \times 10^{3}$ per well were incubated in 96-well plates for 0, 24, 48 or 72 hours and Cell Counting Kit-8 solution (CCK-8; Dojindo Laboratories, Kumamoto, Japan) was added into the well. At 4 hours after CCK-8 incubation, cell viability was measured by reading optical density value at a wavelength of $450 \mathrm{~nm}$.

Wound Healing Assay. Cells were seeded into a six-well culture plate. A scratch wound was created in the center of the cell culture plate with a sterile pipette tip when the cells had reached $90 \%$ confluence. The cells were washed with PBS twice to remove the debris. The medium was replaced with $\alpha$-MEM supplemented with $1 \%$ FBS. Three randomly selected fields along the scraped line were photographed under an inverted microscope. The distance from one side of the scratch to the other was measured using Image Pro-Plus 6.0 software (Media Cybernetics, Rockville, MD).

Invasion Assay. For the migration assays, $5 \times 10^{4}$ cells were seeded into the upper chamber of the Millicell Hanging Cell Culture inserts $(8 \mu \mathrm{m}$ pore size; Millipore, Billerica, MA). For the invasion assays, $1 \times 10^{5}$ cells were seeded into the upper chamber of the insert coated with Matrigel (BD Bioscience, San Jose, CA). Chambers were placed in wells with $\alpha$-MEM containing 10\% FBS. After 24 hours of incubation at $37^{\circ} \mathrm{C}$, the noninvading cells were removed by wiping with a cotton swab. Cells that had migrated or invaded through the filter membrane were fixed and stained with Mayer's hematoxylin (Sigma-Aldrich, St. Louis, MO). The number of invading cells on the membrane was counted in five microscopic fields (original magnification, $400 \times$ ). The experiments were repeated in triplicate.

Flow Cytometry. Cells were harvested, washed with cold PBS, and fixed with $75 \%$ cold ethanol at $4^{\circ} \mathrm{C}$ overnight. After fixation, the cells were collected and incubated with $100 \mu \mathrm{g} / \mathrm{ml}$ RNase A (Sigma-Aldrich) at $37^{\circ} \mathrm{C}$ for 30 minutes, followed by incubation with $25 \mu \mathrm{g} / \mathrm{ml}$ propidium iodide (Sigma-Aldrich) at room temperature in the dark. The cell cycle distribution was analyzed by a fluorescenceactivated cell sorter (FACS) flow cytometer (Becton Dickinson, Franklin Lakes, NJ).

Biotin-Coupled miRNA Capture. Approximately $10^{6}$ cells were transfected with $50 \mu \mathrm{M}$ of biotinylated miR-144 mimic or ROCK1/ RhoA 3'-UTR binding site mutant miR-144 mimic (GenePharma, Shanghai, People's Republic of China) at 50\% confluence for lysis. At 24 hours after transfection, the cells were harvested and washed in PBS, lysed in lysis buffer. A total of $50 \mu \mathrm{l}$ washed streptavidin magnetic beads were blocked for 2 hours and then added to each reaction tube to pull down the biotin-coupled RNA complex. All the tubes were incubated for 4 hours on the rotator at a low speed (10 rpm). The beads were washed with lysis buffer 5 times, and TRIzol LS (Life Technologies, Carlsbad, CA) was used to recover RNAs specifically interacting with miRNA. The abundance of ROCK1 and RhoA 3 '-UTR in bound fractions was evaluated by quantitative reversetranscription polymerase chain reaction (qRT-PCR) analysis.

Dual-Luciferase Reporter Assays. The ROCK1 and RhoA 3'UTR binding sites of miR-144 were predicted by TargetScan (http:// www.targetscan.org/vert_71/). The different fragment sequences were synthesized and then inserted into the pGL3-basic vector (Promega, Madison, WI) by Hanbio (Shanghai, People's Republic of China). All plasmids for transfection were verified by sequencing and prepared using the QIAGEN plasmid purification kit (QIAGEN, Hilden, Germany). HEK293T cells were transiently transfected using Lipofectamine 3000 (Invitrogen, Carlsbad, CA) according to the manufacturer's instructions. Twenty-four hours after transfection, cells were lysed, and Firefly and Renilla luciferase activities were measured using the Dual-Luciferase Reporter Assay System 
(Promega) according to the manufacturer's protocol. Each experiment was repeated at least 3 times.

Lentivirus Production and Infection. The luciferase gene was cut from pGL3-Basic plasmid (Promega) with NheI and XbaI. Plentineo (Invitrogen) was digested with SpeI and dephosphorylated using calf intestinal phosphatase. Then the luciferase gene was subcloned into the SpeI site of the plenti-neo thus resulting in the recombinant expression vector plenti-neo-luc. Purified plenti-neo-luc and the packaging mixture were cotransfected into 293FT cells using Lipofectamin 2000 to generate lentivirus. The concentrated virus then was transduced to SAOS2 cells with $8 \mu \mathrm{g} / \mathrm{ml}$ of polybrene (SigmaAldrich). The corresponding cell line stably expressing luciferase was named SAOS2-luc.

Western Blot Analysis. Whole-cell lysates were prepared with ice-cold lysis buffer (50 mM Tris-HCl, pH 7.4, $150 \mathrm{mM} \mathrm{NaCl}, 1 \%$ NP-40, and $0.1 \%$ sodium dodecyl sulfate) supplemented with protease inhibitors (Complete Tablet; Roche, Mannheim, Germany). The protein concentration was determined using a Pierce BCA Protein Assay Kit (Thermo Fisher Scientific, Houston, TX). The proteins were sizefractionated by SDS-PAGE and transferred to a polyvinylidene difluoride membrane (Hybond-P; Amersham Biosciences, Amersham, United Kingdom). The membranes were incubated overnight at $4^{\circ} \mathrm{C}$ with the primary antibodies. The antibodies against ROCK1, RhoA, and GAPDH (I-19) were obtained from Santa Cruz Biotechnology (Dallas, TX). After washing with Tris-buffered saline containing $0.1 \%$ Tween, the membranes were incubated with horseradish peroxidase-conjugated secondary antibodies for 1 hour at room temperature.

RNA Extraction and Quantitative Real-Time PCR. Total RNA, including miRNA, was extracted using the miRNeasy Mini Kit (QIAGEN) according to the manufacturer's instructions. Then $1 \mu \mathrm{g}$ total RNA was reverse-transcribed with a specific stem-loop primer for miRNA. After the reverse-transcription reaction, real-time PCR was performed by an ABI 7900HT system using SYBR Premix ExTaq (Takara, Madison, WI). Small nuclear RNA U6 was used as an internal control for miRNA. The primer sequences used for real-time PCR are presented in Table 2.

Generation of Osteosarcoma Model. Animal handling and experimental procedures were performed following the ethical guidelines of the Shanghai Jiao Tong University School of Medicine. We anesthetized 4- to 6-week-old male nu/nu athymic mice with tribromoethanol. Human OS SAOS2-luc cells were injected into the tibia $\left(1 \times 10^{7}\right.$ cells in $50 \mu \mathrm{l}$ PBS $)$ of athymic mice to induce a tumor (Tu et al., 2012). Tumor growth was monitored by measuring two perpendicular diameters with digital calipers. Tumor volume was calculated using the formula $V=\left(L \times W^{2}\right) / 2$ where $L$ and $W$ represent the largest and the smallest diameter, respectively.

In Vivo Treatment and Bioluminescence Imaging. In vivo jet-polyethylenimine (jet-PEI; Polyplus-Transfection, IllkirchGraffenstaden, France) was used as a transfection reagent. JetPEI/ miR-144 or jetPEI/negative control complexes with a ratio of 1:7 were prepared in a solution of $10 \% \mathrm{w} / \mathrm{v}$ glucose. One week after injection of the tumor cells, the mice were randomly separated into two groups ( $n=10$ mice per group) and treated by way of multipoint intratumoral injection twice a week, according to the manufacturer's instructions. Tumor growth was monitored over time using an In Vivo Imaging System (IVIS; Xenogen, Alameda, CA).
The mice were anesthetized and injected intraperitoneally with D-luciferin. Imaging was performed with an exposure time of 1 minute. Upon termination of the experiment, mice were sacrificed. Tumors, lungs, and livers were immediately fixed in $4 \%$ paraformaldhyde for paraffin embedding. Sections of $5 \mu \mathrm{m}$ were stained with $\mathrm{H} \& \mathrm{E}$ for immunohistochemical analysis. The lungs were examined microscopically for the presence of OS foci. Also, the tumors were spap-frozen for RNA preparation.

Histology and Immunohistochemistry. Osteosarcomas from patients as well as the model animals were fixed overnight with $4 \%$ paraformaldhyde in PBS, decalcified with $12.5 \%$ EDTA, and then embedded in paraffin. Tissue sections $(5 \mu \mathrm{m})$ were deparaffinized in xylene, serially rehydrated in ethanol, and stained with H\&E. For immunohistochemistry, sections in $10 \mathrm{mM}$ sodium citrate buffer $(\mathrm{pH}$ 6.0 ) were heated in a microwave oven and kept at $95^{\circ} \mathrm{C}$ for 10 minutes. The slides were cooled for 30 minutes at room temperature after antigen unmasking. Endogenous peroxidase activity was blocked with $3 \%$ hydrogen peroxide, followed by rinsing several times in PBS.

After blocking and nonspecific protein binding with 5\% bovine serum albumin in PBS for 30 minutes at room temperature, sections were incubated overnight at $4^{\circ} \mathrm{C}$ with primary antibody against ROCK1 (Santa Cruz Biotechnology) and RhoA (Santa Cruz Biotechnology). The slides were rinsed in PBS and then incubated with secondary antibody (EnVision detection kit, Peroxidase/DAB, Rabbit/mouse; Dako Cytomation, Carpinteria, CA) according to the manufacturer's protocol.

After washing, the slides were stained with 3,3'-diaminobenzidine tetrahydrochloride (EnVision detection kit, Peroxidase/DAB, Rabbit/mouse; Dako Cytomation). Sections were counterstained with Mayer's hematoxylin (Sigma-Aldrich). Staining with normal IgG and staining without primary antibody were also performed as negative controls.

Cell Staining and Injections. SAOS2 OS cells cultured at $80 \%$ density were trypsinized and centrifuged at $1000 \mathrm{rpm}$ for 5 minutes. The supernatant was discarded, and the cells were resuspended in serum-free $\alpha$-MEM medium (GIBCO) to $1 \times 10^{6}$ cells $/ \mathrm{ml}$. We then added $5 \mu \mathrm{l}$ of live cell fluorescence labeling reagent CM-Dil $(4 \mathrm{ng} / \mu \mathrm{l}$ final concentration) and resuspended the cells. Cells stained with CM-Dil were incubated at $37^{\circ} \mathrm{C}$ for 20 minutes and centrifuged at $1500 \mathrm{rpm}$ for 5 minutes at $37^{\circ} \mathrm{C}$. We discarded the supernatant and resuspended the cells 4 to 5 times in $100 \mu \mathrm{l} \mathrm{PBS}$ at $37^{\circ} \mathrm{C}$ to prepare for injecting them into zebrafish embryos.

Zebrafish embryos were anesthetized with tricaine 2 days after fertilization, and the cells labeled with CM-Dil were injected into the yolk sac; the cell density was about $2 \times 10^{5}$ cells/ $\mu$ l. Immediately after the injection, we used fluorescence microscopy (FV1000; Olympus, Tokyo, Japan) for observation and photography. We removed the uninjected cells and dead zebrafish embryos. After injection, we incubated the embryos at $31^{\circ} \mathrm{C}$ for 1 hour then at $35^{\circ} \mathrm{C}$; they were observed and photographed every 24 hours.

Statistical Analysis. The results are expressed as mean \pm S.D. The $P$ values in Table 1 were obtained from Pearson's chi-squared test or Fisher's exact test. The Kaplan-Meier analysis was used to estimate the overall survival, and the log-rank test was used to evaluate the differences between survival curves. The statistical significance of differences between the two groups was calculated by using Student's $t$ test. The other statistical analyses performed were Dunnett's or Tukey-Kramer's tests, as post hoc tests after ANOVA. The details

TABLE 2

Primer sequences used for real-time PCR

\begin{tabular}{|c|c|c|}
\hline & Sense Primer $\left(5^{\prime}-3^{\prime}\right)$ & Antisense Primer $\left(5^{\prime}-3^{\prime}\right)$ \\
\hline miR-144 & GCGTGCTACAGTATAGATGATG & GTGCAGGGTCCGAGGT \\
\hline $\mathrm{U} 6$ & CTCGCTTCGGCAGCACA & AACGCTTCACGAATTTGCGT \\
\hline ROCK1 & AACATGCTGCTGGATAAATCTGG & TGTATCACATCGTACCATGCCT \\
\hline RhoA & TTTGGAGGTGGCATAGCCTT & ATGTTTAGTCAGCTGGAGAGAAGAG \\
\hline GAPDH & ACAACTTTGGTATCGTGGAAGG & GCCATCACGCCACAGTTTC \\
\hline
\end{tabular}


are indicated in figure legends. $P<0.05$ was considered statistically significant.

\section{Results}

MiR-144 Functions as a Tumor Suppressor in Osteosarcoma Cells. To evaluate the role of miR-144 in OS, we first transfected miR-144 mimics or miR-NC (negative control miRNA) into SAOS2 and U2-OS cells. Transfection of miR-144 mimics significantly suppressed the cell proliferation after 72and 96-hours of culture, which was determined by CCK-8 assay (Fig. 1A). To further explore the role of miR-144 in OS cell proliferation, we investigated cell cycle distribution by flow cytometry. We found that the miR-144-mimic transfected cells accumulated at the G0/G1 phase of cell cycle (Fig. 1B).
Soft agar assays showed that miR-144 significantly suppressed the anchorage-independent growth in SAOS2 and U2-OS cells (Fig. 1C).

We also investigated whether miR-144 would cause motility alteration. The results of the invasion and wound-healing assays showed that miR-144 dramatically compromised the invasion and migration abilities of SAOS2 and U2-OS cells (Fig. 1, D and E). Collectively, these results demonstrated that miR-144 functions as a tumor suppressor in OS cells, markedly inhibiting cell growth, migration, and invasion abilities.

ROCK1 and RhoA Are Direct Targets of miR-144. Because miR-144 has been shown to serve multiple biologic functions and especially plays an important role in OS cancer etiology, we investigated which genes are directly regulated by miR-144 in OS. With the help of the miRNA
A

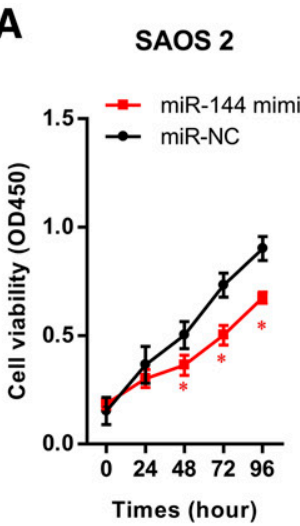

c
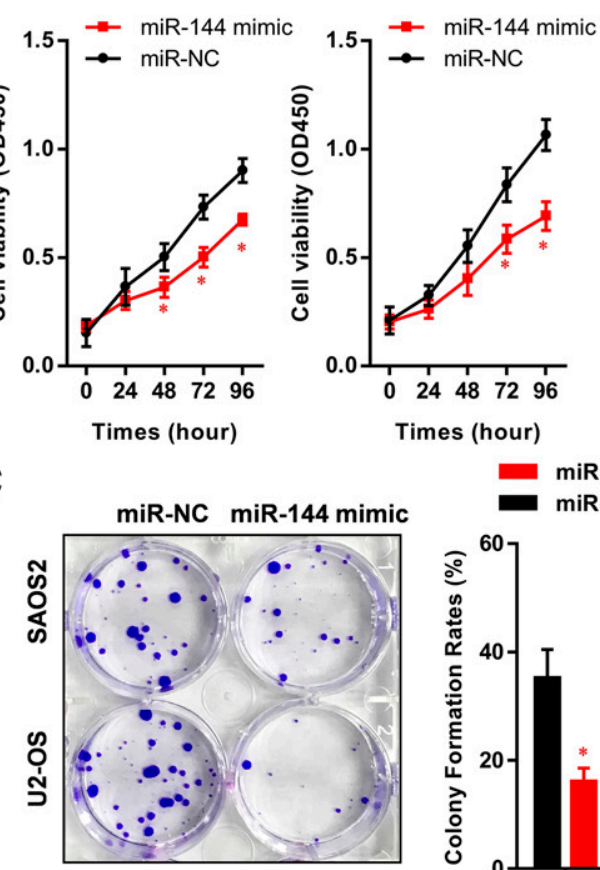

B

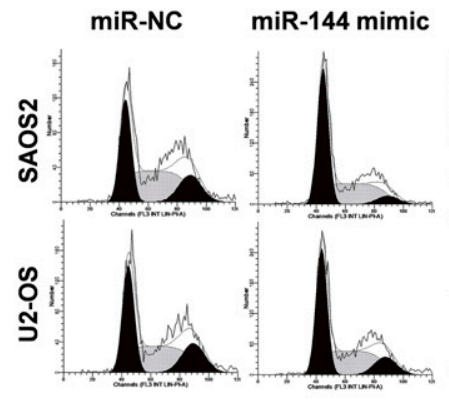

miR-144 mimic

- miR-NC
miR-144 mimic

- miR-NC

E

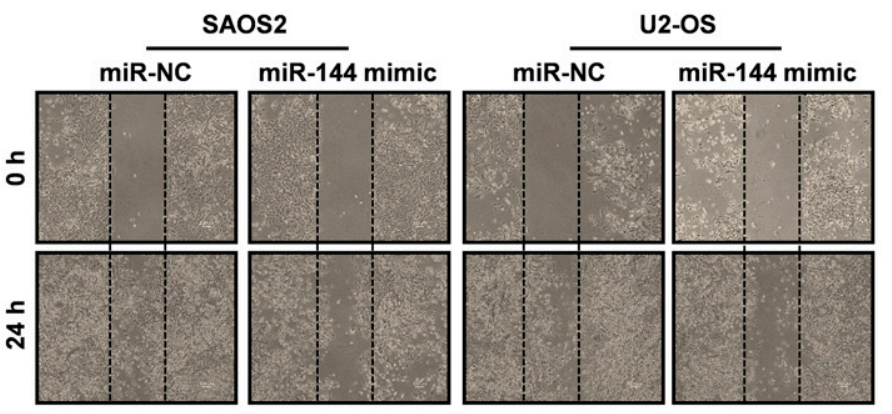

miR-144 mimic

D
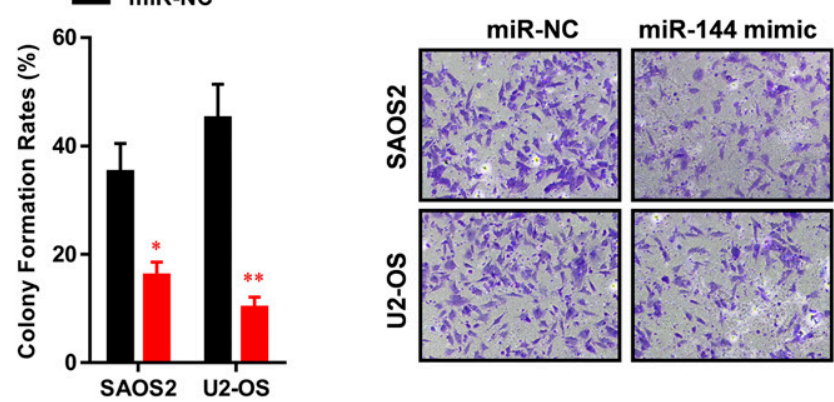

miR-NC
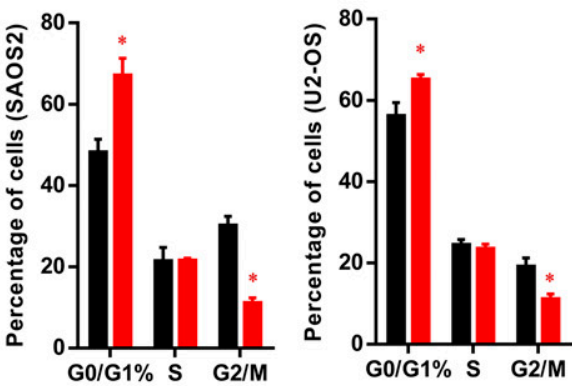

miR-144 mimic

miR-NC

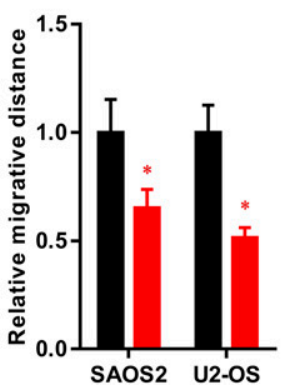

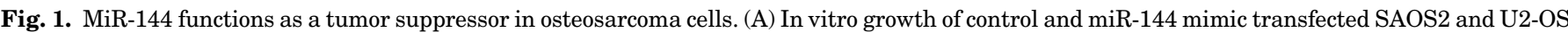

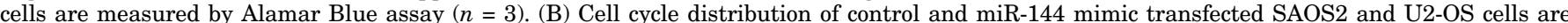

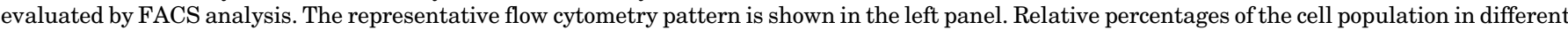

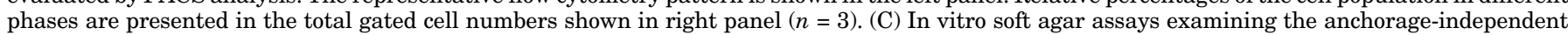

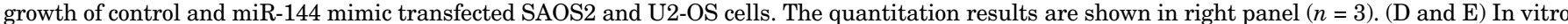

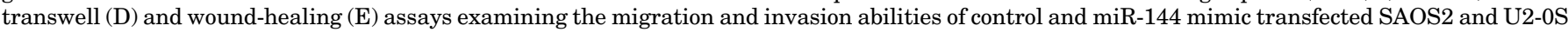

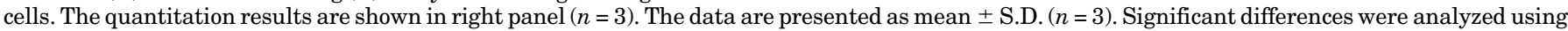
Student's $t$ test compared with the miR-NC group and are marked with asterisks $(* P<0.05 ; * * P<0.01)$. 
target prediction databases TargetScan (Lewis et al., 2005) (http://www.targetscan.org) and miRanda (Betel et al., 2008) (http://www.microrna.org), we predicted that miR-144 would bind to RhoA and its pivotal downstream effector ROCK1 mRNA, and we performed further analyses.

To determine whether miR-144 regulated RhoA and ROCK1 expression, we examined the protein levels of RhoA and ROCK1 in control and miR-144 mimics transfected SAOS2 and U2-OS cells. Our results demonstrated that RhoA and ROCK1 protein were significantly decreased in miR-144mimic transfected SAOS2 and U2-OS cells (Fig. 2A). The pulldown assay also revealed more enrichment of ROCK1 and RhoA 3'-UTR in the miR-144-captured fraction when compared with the introduction of a miR-144 mutation that disrupted the binding site of miR-144 for ROCK1/RhoA 3'UTR (Fig. 2B).

Moreover, we performed anti-AGO2 RNA immunoprecipitation and examined the miR-144, RhoA and ROCK1 mRNA expression. Our results showed that miR-144 was enriched in AGO2-containing complexes, and miR-144 bound to RhoA and ROCK1 mRNA (Fig. 2C).

To further validate the computational finding that miR-144 negatively regulated RhoA and ROCK1, RhoA and ROCK1 3'UTR containing the wild-type or mutant miR-144 binding site were cloned into the psiCHECHK-2 plasmid (Fig. 2D, left). We found that miR-144 mimics significantly repressed the luciferase reporter activity of the wild-type $3^{\prime}$-UTR whereas the luciferase reporter activity of the mutant $3^{\prime}$-UTR was not influenced by miR-144 (Fig. 2D, right). These results demonstrated that RhoA and ROCK1 are direct targets of miR-144.

MiR-144 Inhibits Osteosarcoma Cells Proliferation through Dual-Inhibition of RhoA 1 and ROCK. Having established the tumor-suppressive role of miR-144 and verified its direct targets RhoA and ROCK1, we investigated how miR-144 inhibited OS tumorigenesis via RhoA/ROCK1 pathway. We transfected ROCK1 and RhoA respectively or cotransfected ROCK1 and RhoA in miR-144 transfected SAOS2 and U2-OS cells using adenovirus infection (Fig. 3A). Our results suggested that cell growth, migration, and invasion abilities were equally significantly increased after individual RhoA overexpression and RhoA and ROCK1 co-overexpression in miR-144 transfected SAOS2 and U2-OS cells; individual overexpression of ROCK1 had no statistical significance compared with controls in miR-144 transfected SAOS2 and U2-OS cells (Fig. 3, B-E). These results suggested that miR-144 exerted antitumor effects through dual-inhibition of the RhoA/ROCK1 pathway. Only ROCK1 rescuing failed to diminish the antitumor effects of miR-144 in OS cells.
A

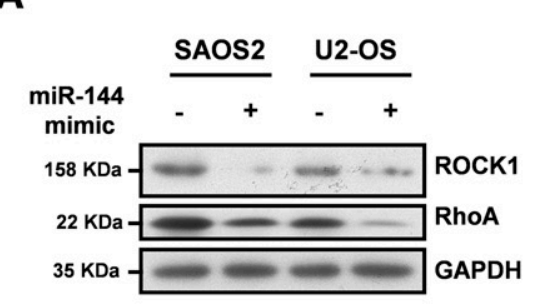

C
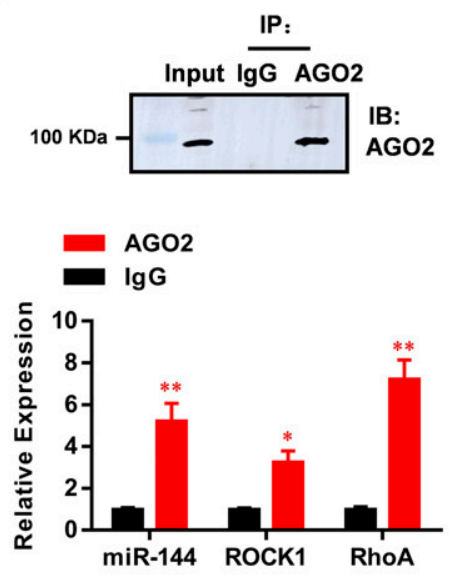

miR-144 mimic

miR-NC

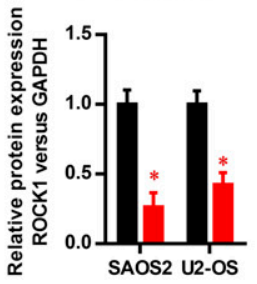

D

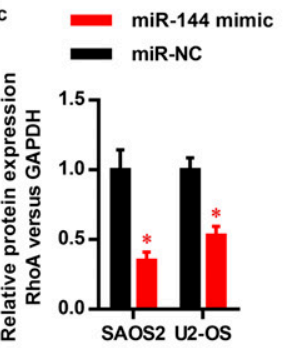

ROCK1/RhOA IRNA

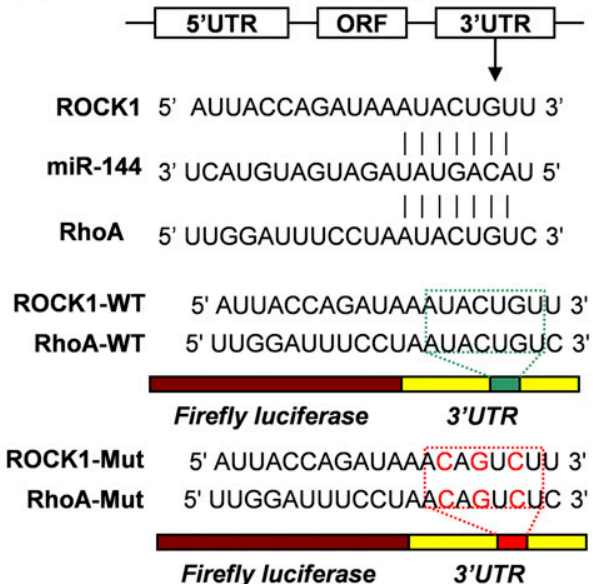

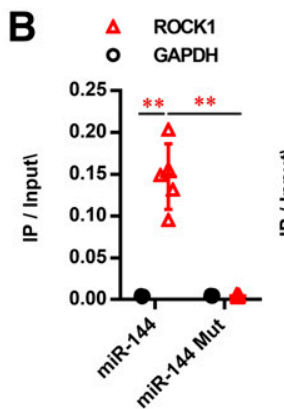
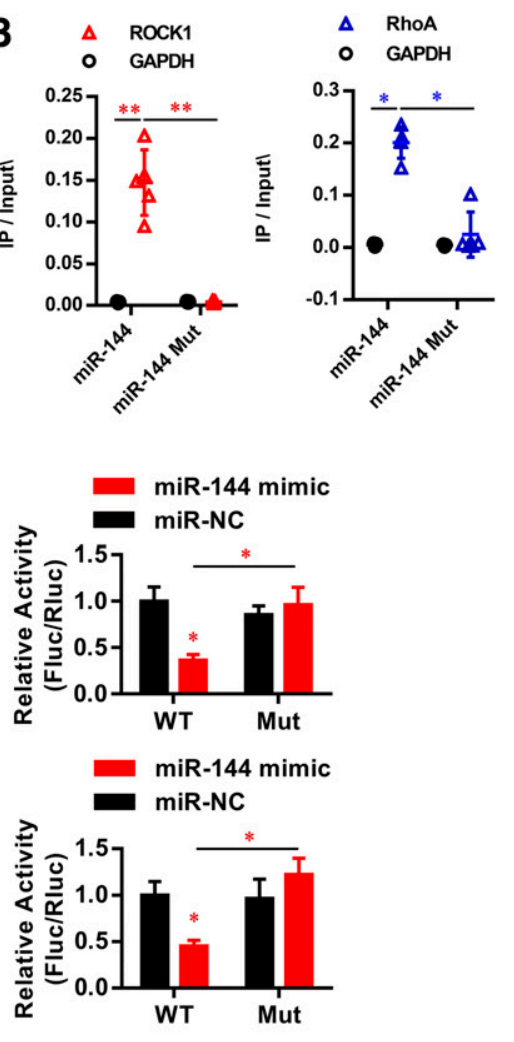

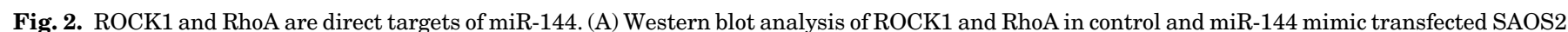

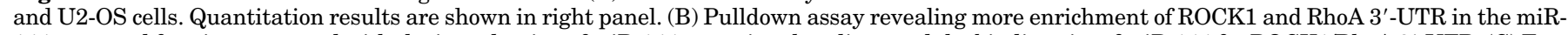

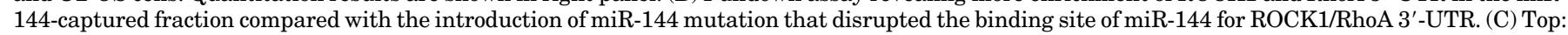

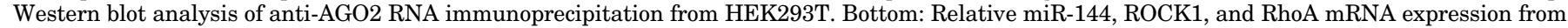

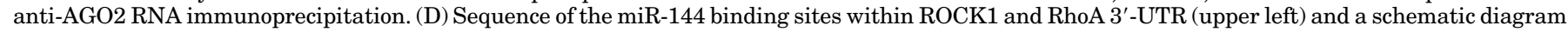

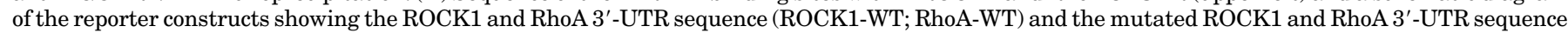

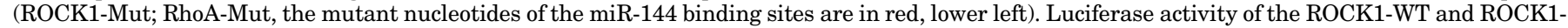

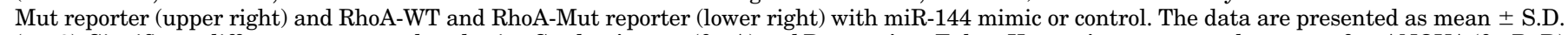

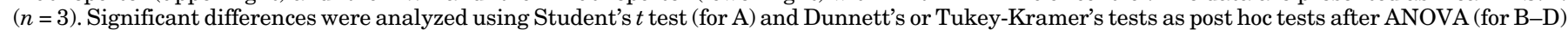
and are marked with asterisks $(* P<0.05 ; * * P<0.01)$. 
A

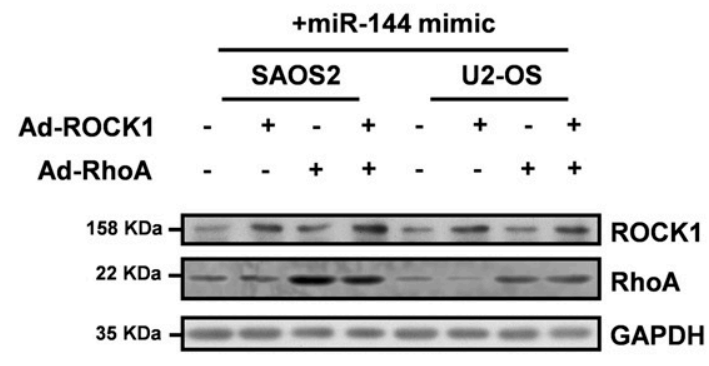

B

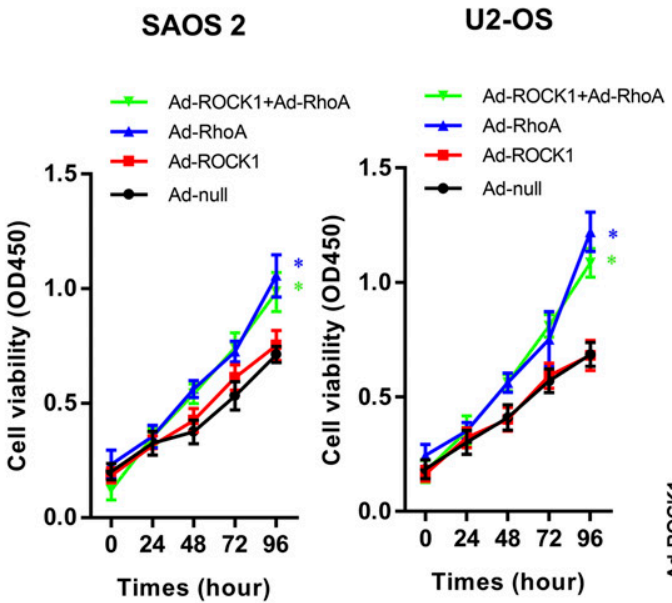

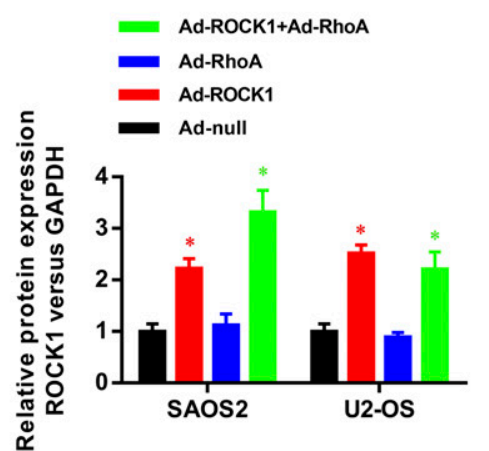

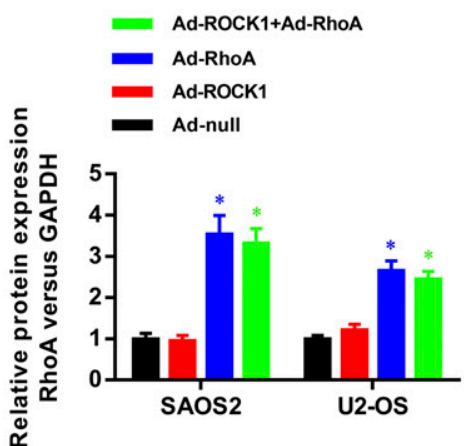

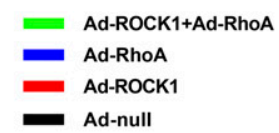

Ad-ROCK1+Ad-RhoA
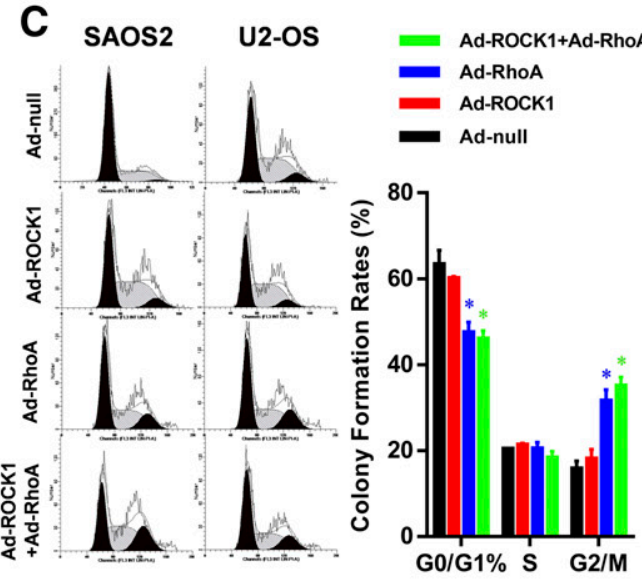

Ad-ROCK1

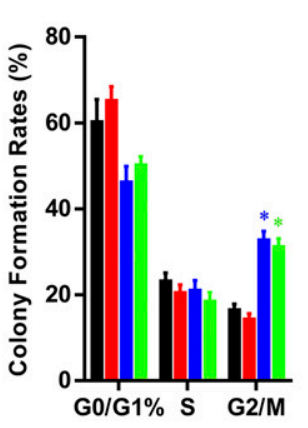

D
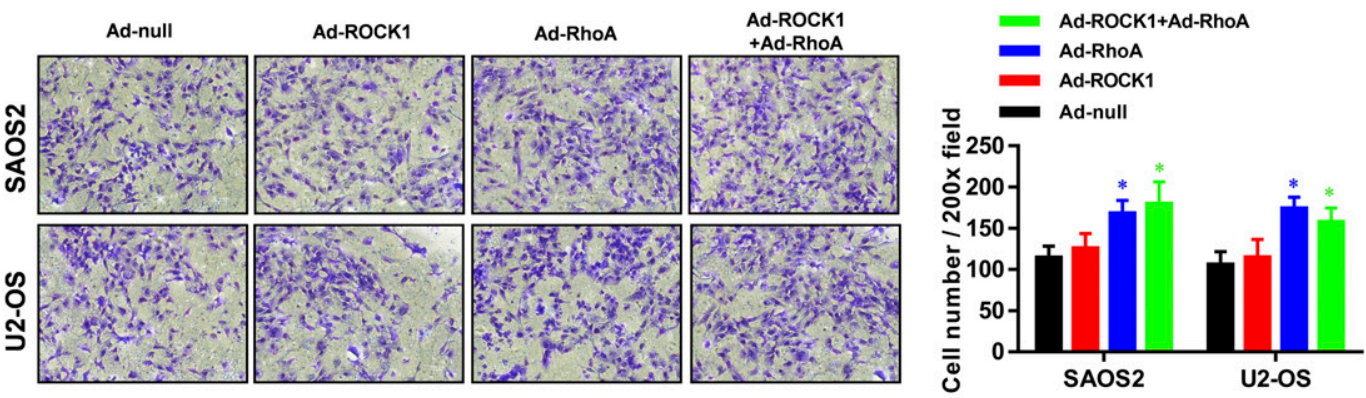

E
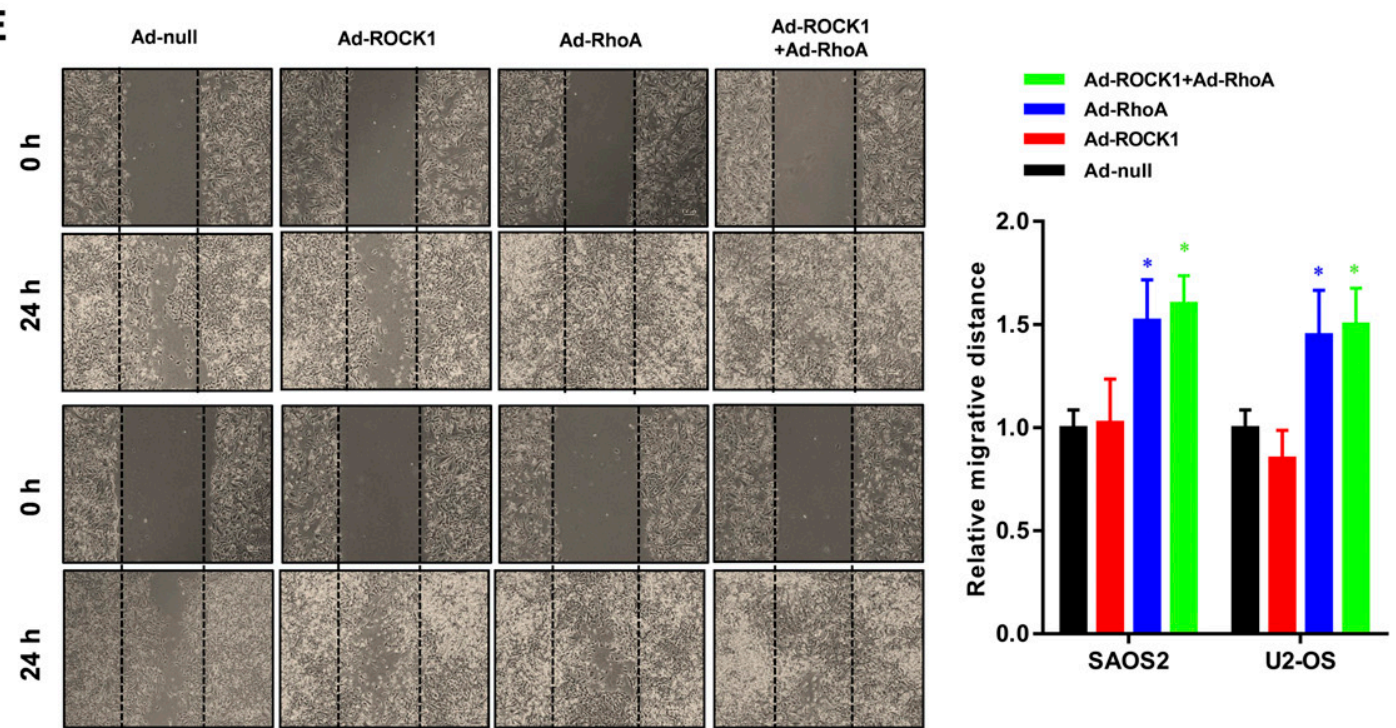

Fig. 3. MiR-144 regulates ROCK1 and RhoA to inhibit osteosarcoma tumorigenesis. (A) Western blot analysis of ROCK1 and RhoA in miR-144 mimic transfected SAOS2 and U2-OS cells with control and ROCK1, RhoA, or both transfection. Quantification results are shown in the right panel. (B) Left: In vitro growth of miR-144 mimic transfected SAOS2 and U2-OS cells with control and ROCK1, RhoA, or both transfection $(n=3)$. (C) FACS analyses showing cell cycle distribution of miR-144 mimic transfected SAOS2 and U2-OS cells with control and ROCK1, RhoA, or both transfection $(n=3)$. (D and E) 
A

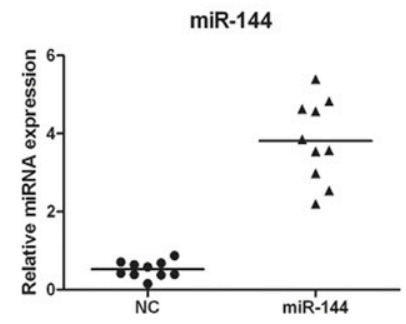

C

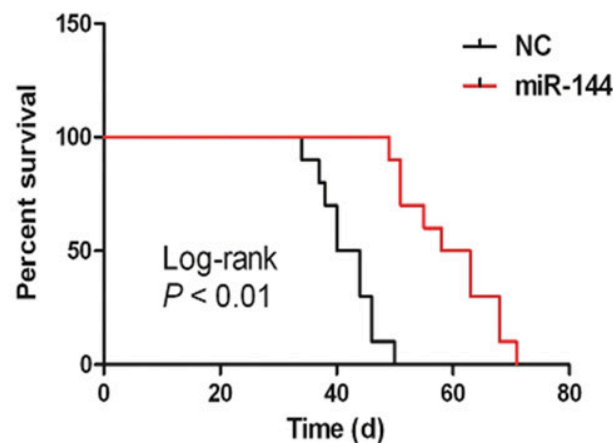

Time (d)
B

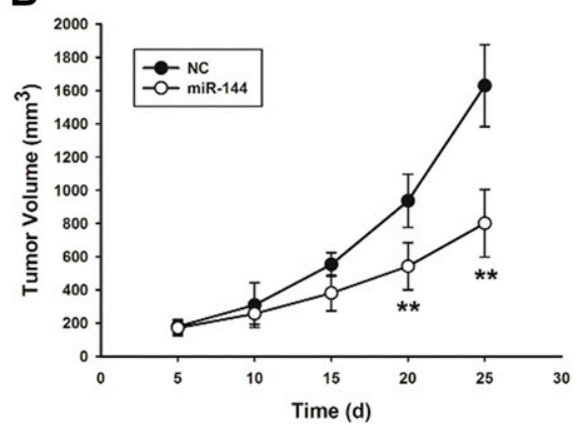

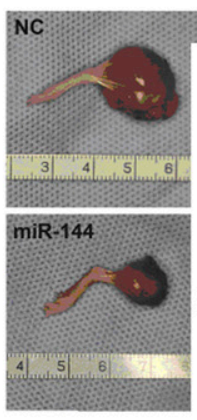

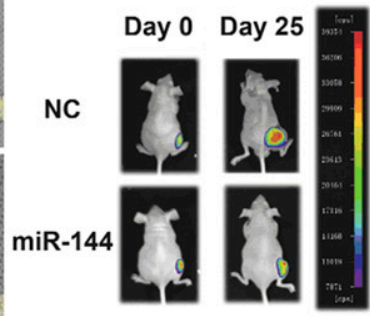

D

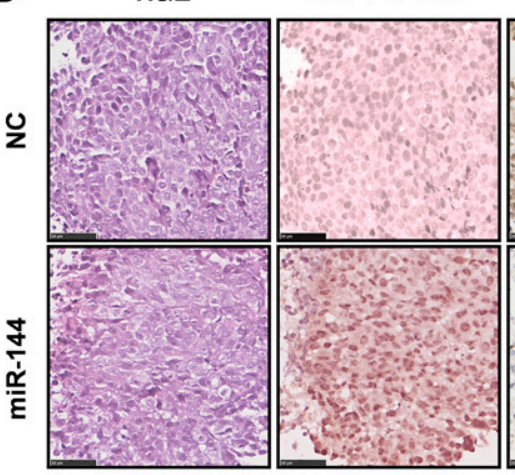

ROCK1

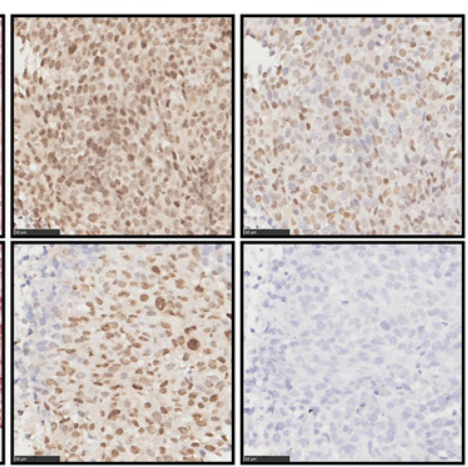

Fig. 4. MiR-144 inhibits tumorigenesis and metastasis in vivo. (A) The elevated miR-144 level in the xenograft tumors was confirmed by qRT-PCR. (B) Left: tumor volume of xenografts in nude mice injected SAOS2 cells with control or miR-144 oligonucleotides treatment $(n=10)$. Middle: representative images of tumors treated with NC or miR-144. Right: representative in vivo imaging photos of NOD mice treated with NC or miR-144. (C) Animal survival of tumorigenesis analysis of SAOS2 cells with NC or miR-144 treatment in nude mice. (D) H\&E, miRNA-144 in situ hybridization (ISH) and RCOK1/ RhoA IHC stained images of tibia sections from NC and miR-144-treated mice. The data are presented as mean \pm S.D. $(n=3)$. Significant differences (in B) were analyzed using Dunnett's or Tukey-Kramer's tests as post hoc tests after ANOVA (for B-D) and are marked with asterisks (** $P<0.01$ ). KaplanMeier analysis (in C) was used to estimate the overall survival, and the log-rank test was used to evaluate the differences between survival curves.

MiR-144 Inhibits Tumorigenesis and Metastasis In Vivo. To further confirm the tumor-suppressive role of miR-144 in vivo, we then validated our hypothesis in the mouse and zebrafish models. Tumorigenic human OS cells SAOS2, stably expressing Firefly luciferase, were injected into the tibia of nude mice. After the tumor xenografts had been established, the mice were intratumorally injected with either miR-144 or NC oligonucleotides complexed twice a week, and the tumor growth was monitored by bioluminescence imaging.

Overexpression of miR-144 dramatically reduced the tumor volume of the xenografts in nude mice (Fig. 4, A and B). Median survival was shortened by about 2.6 weeks in the NC group compared with the miR-144-treated mice, as assessed by Kaplan-Meier curves (Fig. 4C). In situ hybridization and immunohistochemical analysis of tumor sections clearly demonstrated the reduced expression of ROCK1/RhoA in the miR144-elevated mice (Fig. 4D).

In addition, human OS cells SAOS2 stained with CM-Dil were injected into the yolk sac of zebrafish 2 days after fertilization, comprising the hybrid offspring of miR-144 knockout zebrafish and wild-type zebrafish. Marked zebrafish were observed every 24 hours and photographed by laser scanning confocal microscopy. After 24 hours, OS cells started to proliferate and obvious metastasis was observed. After 48 hours, the miR-144 knockout zebrafish showed significant tumor cell proliferation and metastasis compared with wild-type zebrafish. Metastasis to heart, liver, and other organs was obviously observed, and noticeable tumor cell masses were enriched in blood islands in miR-144 knockout zebrafish (Fig. 5, D-F) while wild-type zebrafish showed no apparent tumor metastasis (Fig. 5, A-C). Thus, these findings revealed that miR-144 inhibited tumorigenesis and metastasis in vivo.

Clinical Relevance of miR-144 and ROCK1 in Osteosarcoma Patients. We next assessed whether the relationship of miR-144 and RhoA/ROCK1 could be recapitulated in human OS. Patients who were histologically confirmed as having OS were enrolled at Shanghai Ninth People's Hospital. Table 1 summarized the relation between the expression of miR-144 and the characteristics of the OS patients. Most tumors displayed osteoblastic histology (82.4\%). The level of miR-144 was significantly down-regulated in the patients who developed metastasis during the follow-up period.

In vitro transwell (D) and wound-healing (E) assays examining the migration and invasion abilities of miR-144 mimic transfected SAOS2 and U2-OS cells with control and ROCK1, RhoA, or both transfection. The data are presented as mean \pm S.D. $(n=3)$. Significant differences were analyzed using Dunnett's or Tukey-Kramer's tests as post hoc tests after ANOVA (for B-D) and are marked with asterisks $\left({ }^{*} P<0.05\right.$ ). 
NC

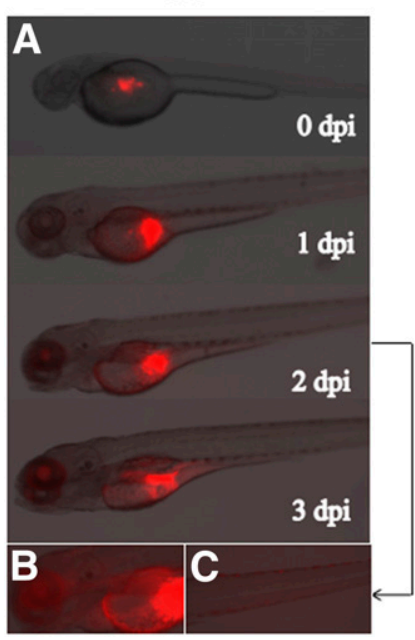

-/- miR-144

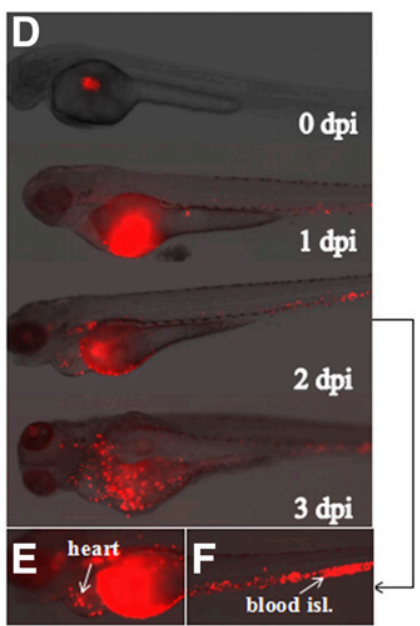

Fig. 5. MiR-144 inhibits tumor metastasis in vivo. Images of wild-type and miR-144 knockout zebrafish injected with CM-Dil stained SAOS2 cells showing the tumorigenic progression and metastasis to heart and blood islands at 2 days postinjection (dpi) $(n=3)$.

Because increased ROCK1 expression has been reported to contribute to OS progression (Wang et al., 2013), we examined miR-144, RhoA, and ROCK1 expression in OS tissue samples and normal human bone fragments by immunohistochemistry. Our results showed that the OS specimens had a decreased miR-144 level (Fig. 6A) and elevated level of RhoA/ROCK1 expression (Fig. 6B) relative to normal osteoblasts. An inverse correlation $(R=-0.758)$ between RhoA/ ROCK1 and miR-144 found in OS specimens (Fig. 6C) further suggested that miR-144 might be involved in the progression of RhoA/ROCK1. Furthermore, we found much lower miR-144 expression in three human OS cell lines-SAOS2, MG63, and U2-OS-compared with the human osteoblastic cell line hFOB1.19 (Fig. 6D). The RhoA/ROCK1 mRNA and protein levels were elevated in all three OS cell lines (Fig. 6, D and E).

Taken together, these data thus suggest that the antioncogenic function of miR-144 may be largely dependent on the dualsuppressive regulation of RhoA and ROCK1 expression in the malignant progression of OS.

\section{Discussion}

Previous studies have shown that ROCK1 is frequently overexpressed in multiple categories of cancer, particularly in metastatic tumors. Increased expression of RhoA and ROCK1 is a prognostic indicator associated with metastasis and poor survival in esophageal (Wang et al., 2010), breast (Patel et al., 2012), and bladder (Kamai et al., 2003) cancers. During tumor invasion and metastasis, cell migration mediated by actinmyosin contraction is one of the key steps. Rho GTPases are critical regulators of actin cytoskeleton and cell migration.

RhoA is one of the major Rho GTPases involved in these processes. ROCK1 is activated when its $\mathrm{C}$-terminal region binds to RhoA and thus induces the formation of stress fiber (Amano et al., 1997) and focal adhesion (Ishizaki et al., 1997) by the phosphorylation of myosin light chain at serine 19 (Ser19). In addition, ROCK1 phosphorylates and activates LIMK, which in turn phosphorylates and inactivates the actin depolymerization factor cofilin. Small interfering
RNA-mediated knockdown of ROCK1 causes reorganization of cytoskeleton and decreased cell mobility (Liu et al., 2009). And overexpression of a dominant-negative ROCK1 construct or the ROCK-specific inhibitor 4 -[(1 \{R\})-1-aminoethyl]- $\{N\}$-pyridin-4-ylcyclohexane-1-carboxamide (Y-27632) reduces tumor cell invasion (Takamura et al., 2001).

In the current study, we observed that ROCK1 expression was relatively higher in OS specimens compared with human normal bone, which is consistent with previous reports. Importantly, we found that the expression of ROCK1 and RhoA were regulated by miR-144 in OS cell lines.

Accompanied by the up-regulation of ROCK1, miR-144 expression is decreased in OS specimens and cell lines. Both miR-144 and miR-451 are transcribed from the same gene locus on human chromosome 17 . However, because of the sequence-specific posttranscriptional modulation, the expression levels of miR-144 and miR-451 are different in cells (Rasmussen et al., 2010). Down-regulation of miR-144 is reported in various cancers (Wang et al., 2011), suggesting that decreased miR-144 levels are not tumor-type specific; thereby, miR-144 may play an important role in tumorigenesis and tumor progression. Son et al. (2009) performed miRNA microarray analysis and found that miR-144 was downregulated in non-small-cell lung cancer. Decreased expression of miR-144 was also found to be correlated with the progression of colorectal cancer (Iwaya et al., 2012). More recently, Namløs et al. (2012) reported that miR-144 was found to be down-regulated in OS.

However, the exact function of miR-144 in cancer has yet to be well elucidated. The tumor-suppressive role of miR-144 by targeting ROCK1 in OS has been previously reported (Cai et al., 2015; Wang et al., 2015). However, no investigators have reported on the rescue effect of ROCK1. It seems that ROCK1 rescue is not work. In this study, we identified a miR-144binding site in both ROCK1 and RhoA 3 '-UTR by in silico analysis. We demonstrated that ROCK1 and RhoA are direct targets of miR-144 and overexpression of miR-144 could repress the expression of ROCK1 and RhoA protein by posttranscriptional regulation. In addition, miR-144 led to a reduction of cell proliferation by blocking the cell cycle at G0/ G1 phases and significantly inhibited cell migration and invasion. The tumor-suppressive role of miR-144 was also confirmed in mouse and zebrafish models.

The therapeutic use of miRNA depends on systemic and/or local application of a specific miRNA-inducing reagent in vivo. We complexed synthetic miR-144 with a commercially available in vivo jet-PEI that has been efficient in delivering miRNAs in vivo (Xu et al., 2011). During our investigation, the in vivo administration of miR-144 for 4 weeks greatly repressed tumor growth in an OS xenograft mouse model. Immunohistochemical analysis showed that the ROCK1 expression level of OS in miR-144-treated mice was much lower than in the control mice, indicating miR-144 could mediate ROCK1 expression in vivo. Also, miR-144 prolonged the overall survival time of tumor-bearing mice, which may have resulted from the antiproliferative effect of miR-144 on tumor cells. More importantly, miR-144 led to a substantial reduction in the dimensions and number of lung nodules in our xenograft model. As this mouse model recapitulates many features of human OS, we may speculate that the suppressive effects of miR-144 on tumor growth and lung metastasis may be beneficial for OS patients. 
A

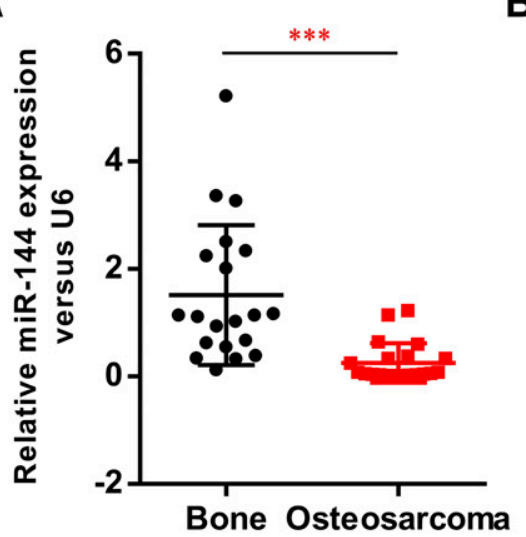

C

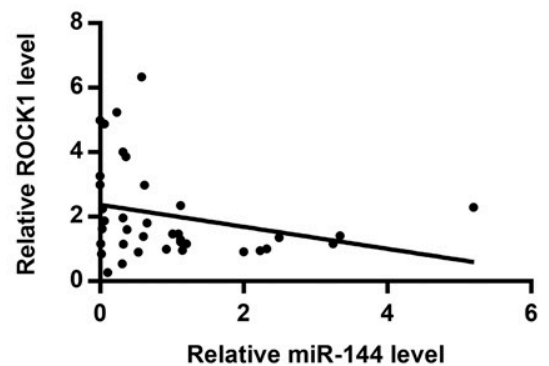

B

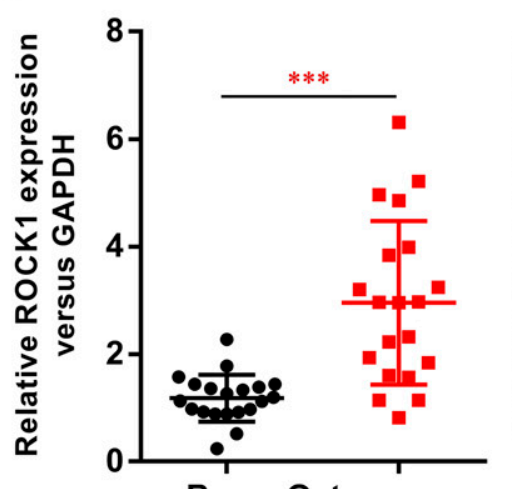

Bone Osteosarcoma

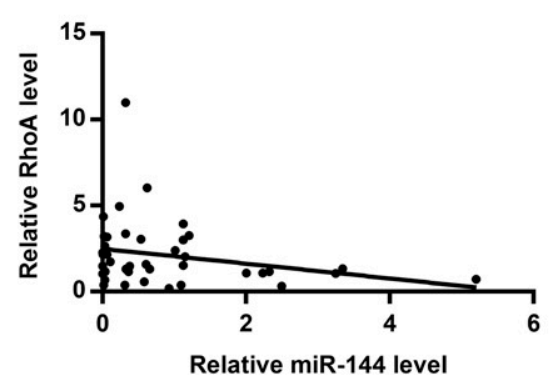

D
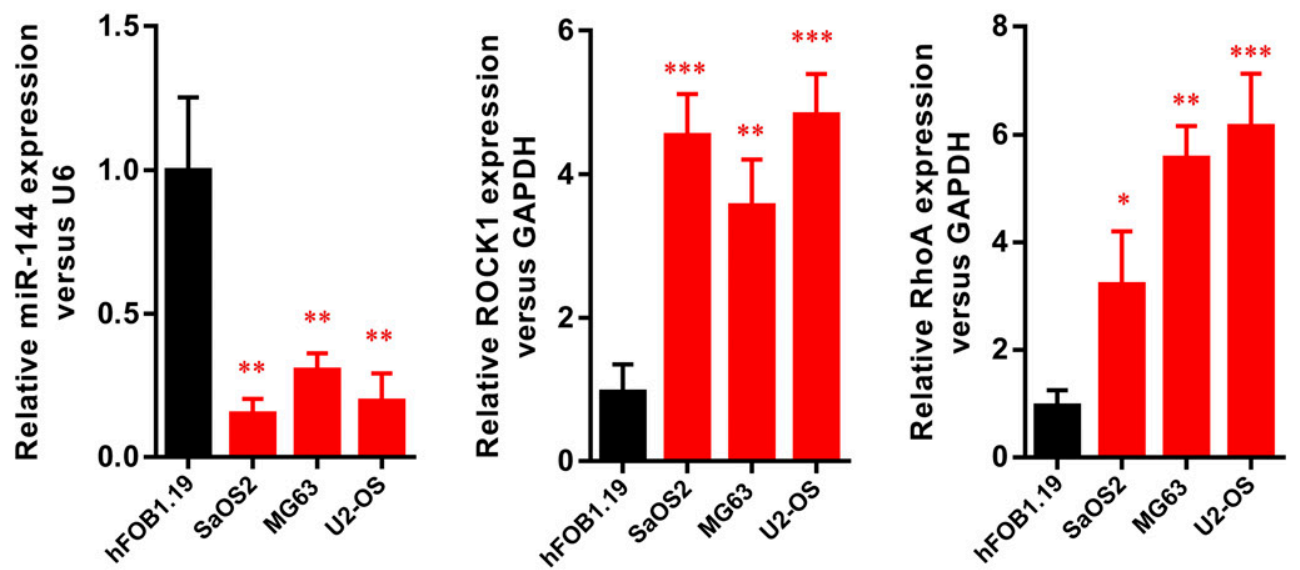

$\mathbf{E}$
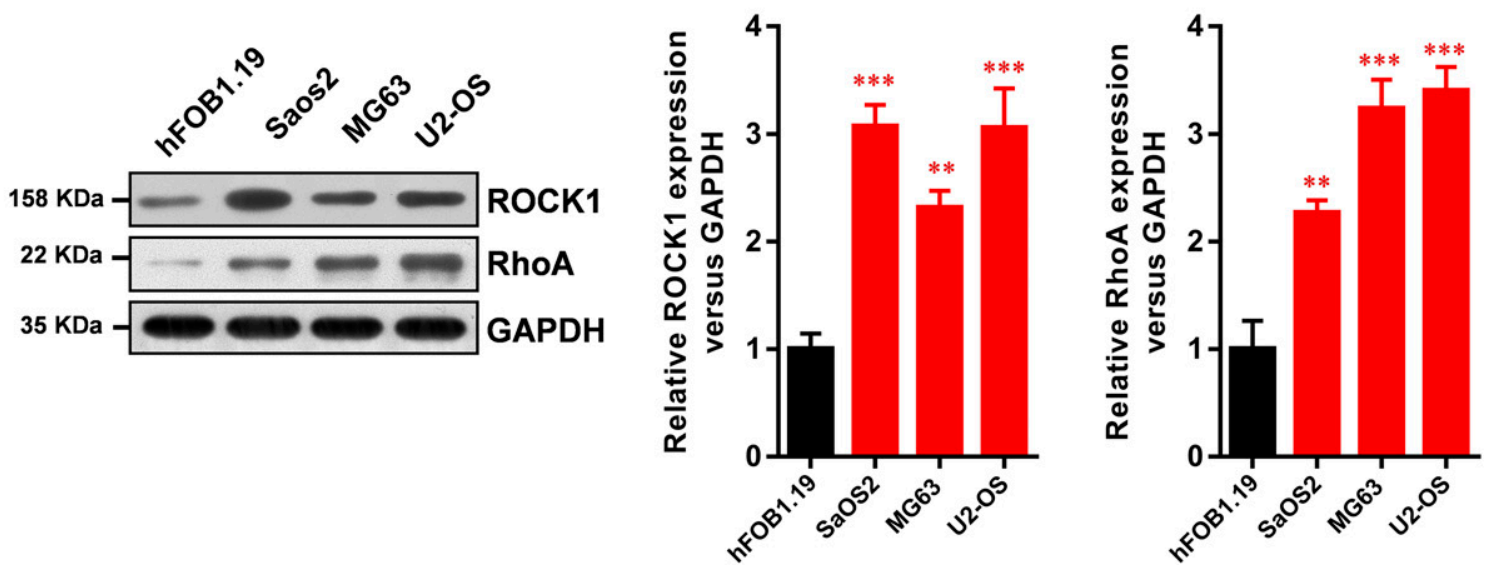

Fig. 6. Clinical relevance of miR-144 and RhoA/ROCK1 in osteosarcoma patients. Real-time PCR analysis of miR-144 (A) and RhoA and ROCK1 (B) in normal human bone and osteosarcoma. (C) Correlation between miR-144 and ROCK1 and RhoA analyzed in normal human bone and osteosarcoma. (D) Real-time PCR analysis of miR-144, ROCK1, and RhoA in human osteoblastic cell line hFOB1.19 and osteosarcoma cell lines SAOS2, MG63, and U2-OS. (E) Western blot analysis of ROCK1 and RhoA in human osteoblastic cell line hFOB1.19 and osteosarcoma cell lines SAOS2, MG63, and U2-OS. The data are presented as the mean \pm S.D. $(n=3)$. Significant differences were analyzed using Student's $t$ test (for A and B) and Dunnett's or Tukey-Kramer's tests as post hoc tests after ANOVA (for D and E). $* P<0.05 ; * * P<0.01 ; * * * P<0.001$. The correlation analysis (in C) used Pearson's chi-squared test. 


\section{8 88888888888888888888888888888888}

Cytosol

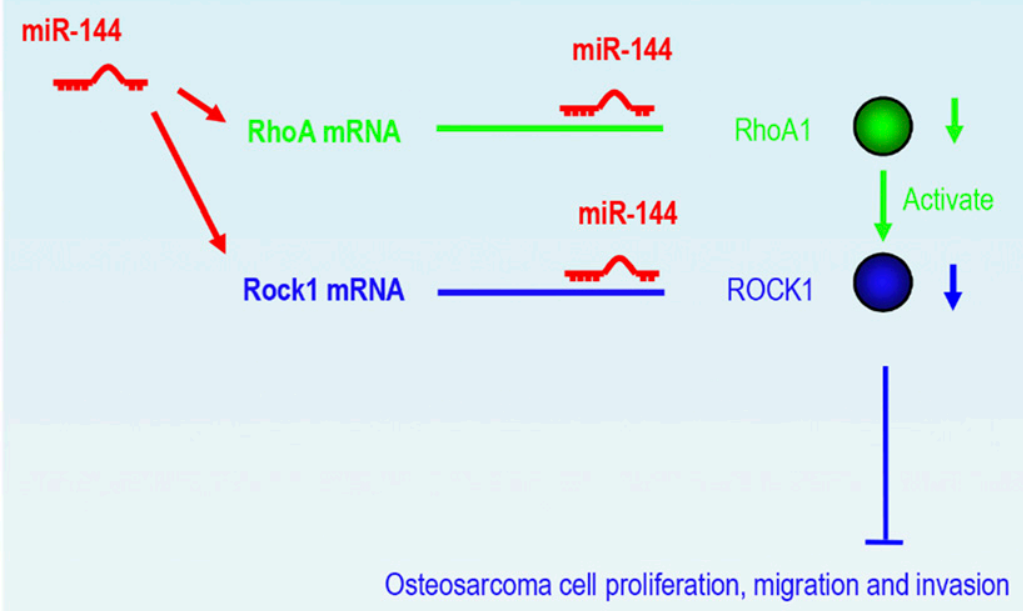

Fig. 7. Model of miR-144 in osteosarcoma tumorigenesis. MiR-144 inhibits tumor growth and metastasis in osteosarcoma via dual-targeting RhoA and its downstream effector ROCK1.
In conclusion, our findings have demonstrated that miR-144 plays a crucial role as a negative upstream regulator of RhoA and its downstream effector ROCK1 through directly targeting their 3'-UTR, inhibiting OS tumor cell proliferation, migration, and invasion (Fig. 7). During OS progression, the attenuation of miR-144 expression may be one of the major causes of the up-regulation of ROCK1, which ultimately stimulates tumor growth and metastasis. Because miR-144 functions as a tumor suppressor for OS, the administration of miR-144 could potentially be a promising therapeutic strategy for treatment of OS.

\section{Authorship Contributions}

Participated in research design: Liu, Zhang.

Conducted experiments: $\mathrm{Liu}, \mathrm{Li}, \mathrm{Xu}$.

Performed data analysis: Liu, Xiao, Cui, Qiao, Chen, Tao.

Wrote or contributed to the writing of the manuscript: Liu, Zhang.

Note Added in Proof-Dr. Wei Dong Tao was added to the byline, author footnotes, and authorship contributions section after publication of the Fast Forward version published on January 23, 2019. In addition, information in the funding footnote was modified from that given in the Fast Forward version.

\section{References}

Alvarez-Garcia I and Miska EA (2005) MicroRNA functions in animal development and human disease. Development 132:4653-4662.

Amano M, Chihara K, Kimura K, Fukata Y, Nakamura N, Matsuura Y, and Kaibuchi K (1997) Formation of actin stress fibers and focal adhesions enhanced by Rhokinase. Science 275:1308-1311.

Bartel DP (2004) MicroRNAs: genomics, biogenesis, mechanism, and function. Cell 116:281-297.

Betel D, Wilson M, Gabow A, Marks DS, and Sander C (2008) The microRNA.org resource: targets and expression. Nucleic Acids Res 36:D149-D153.

Bielack SS, Carrle D, Hardes J, Schuck A, and Paulussen M (2008) Bone tumors in adolescents and young adults. Curr Treat Options Oncol 9:67-80.

Cai SD, Chen JS, Xi ZW, Zhang LJ, Niu ML, and Gao ZY (2015) MicroRNA-144 inhibits migration and proliferation in rectal cancer by downregulating ROCK-1. Mol Med Rep 12:7396-7402.

Cimino D, De Pittà C, Orso F, Zampini M, Casara S, Penna E, Quaglino E, Forni M, Damasco C, Pinatel E, et al. (2013) miR148b is a major coordinator of breast cancer progression in a relapse-associated microRNA signature by targeting ITGA5, ROCK1, PIK3CA, NRAS, and CSF1. FASEB $J$ 27:1223-1235.

Croce CM (2009) Causes and consequences of microRNA dysregulation in cancer. Nat Rev Genet 10:704-714.
Divakaran V and Mann DL (2008) The emerging role of microRNAs in cardiac remodeling and heart failure. Circ Res 103:1072-1083.

Hahmann C and Schroeter T (2010) Rho-kinase inhibitors as therapeutics: from pan inhibition to isoform selectivity. Cell Mol Life Sci 67:171-177.

Harting MT and Blakely ML (2006) Management of osteosarcoma pulmonary metastases. Semin Pediatr Surg 15:25-29.

Hurst DR, Edmonds MD, and Welch DR (2009) Metastamir: the field of metastasisregulatory microRNA is spreading. Cancer Res 69:7495-7498.

Ishizaki T, Naito M, Fujisawa K, Maekawa M, Watanabe N, Saito Y, and Narumiya S (1997) p160ROCK, a Rho-associated coiled-coil forming protein kinase, works downstream of Rho and induces focal adhesions. FEBS Lett 404:118-124.

Iwaya T, Yokobori T, Nishida N, Kogo R, Sudo T, Tanaka F, Shibata K, Sawada G, Takahashi Y, Ishibashi M, et al. (2012) Downregulation of miR-144 is associated with colorectal cancer progression via activation of mTOR signaling pathway. Carcinogenesis 33:2391-2397.

Jianwei Z, Fan L, Xiancheng L, Enzhong B, Shuai L, and Can L (2013) MicroRNA 181a improves proliferation and invasion, suppresses apoptosis of osteosarcoma cell. Tumour Biol 34:3331-3337.

Kamai T, Tsujii T, Arai K, Takagi K, Asami H, Ito Y, and Oshima H (2003) Significant association of Rho/ROCK pathway with invasion and metastasis of bladder cancer. Clin Cancer Res 9:2632-2641.

Leung T, Chen XQ, Manser E, and Lim L (1996) The p160 RhoA-binding kinase ROK alpha is a member of a kinase family and is involved in the reorganization of the cytoskeleton. Mol Cell Biol 16:5313-5327.

Lewis BP, Burge CB, and Bartel DP (2005) Conserved seed pairing, often flanked by adenosines, indicates that thousands of human genes are microRNA targets. Cell 120:15-20.

Lin SL, Chiang A, Chang D, and Ying SY (2008) Loss of mir-146a function in hormone-refractory prostate cancer. RNA 14:417-424.

Link MP, Goorin AM, Miser AW, Green AA, Pratt CB, Belasco JB, Pritchard J, Malpas JS, Baker AR, Kirkpatrick JA, et al. (1986) The effect of adjuvant chemotherapy on relapse-free survival in patients with osteosarcoma of the extremity. $N$ Engl J Med 314:1600-1606.

Liu S, Goldstein RH, Scepansky EM, and Rosenblatt M (2009) Inhibition of rhoassociated kinase signaling prevents breast cancer metastasis to human bone. Cancer Res 69:8742-8751.

Liu X, Choy E, Hornicek FJ, Yang S, Yang C, Harmon D, Mankin H, and Duan Z (2011) ROCK1 as a potential therapeutic target in osteosarcoma. J Orthop Res 29 : $1259-1266$

Lu J, Getz G, Miska EA, Alvarez-Saavedra E, Lamb J, Peck D, Sweet-Cordero A Ebert BL, Mak RH, Ferrando AA, et al. (2005) MicroRNA expression profiles classify human cancers. Nature 435:834-838.

Maekawa M, Ishizaki T, Boku S, Watanabe N, Fujita A, Iwamatsu A, Obinata T, Ohashi K, Mizuno K, and Narumiya S (1999) Signaling from Rho to the actin cytoskeleton through protein kinases ROCK and LIM-kinase. Science 285:895-898.

Mirabello L, Troisi RJ, and Savage SA (2009) Osteosarcoma incidence and survival rates from 1973 to 2004: data from the surveillance, epidemiology, and end results program. Cancer 115:1531-1543.

Nakagawa O, Fujisawa K, Ishizaki T, Saito Y, Nakao K, and Narumiya S (1996) ROCK-I and ROCK-II, two isoforms of Rho-associated coiled-coil forming protein serine/threonine kinase in mice. FEBS Lett 392:189-193.

Namløs HM, Meza-Zepeda LA, Barøy T, Østensen IH, Kresse SH, Kuijjer ML, Serra M, Bürger H, Cleton-Jansen AM, and Myklebost O (2012) Modulation of the osteosarcoma expression phenotype by microRNAs. PLoS One 7:e48086. 
Ohashi K, Nagata K, Maekawa M, Ishizaki T, Narumiya S, and Mizuno K (2000) Rho-associated kinase ROCK activates LIM-kinase 1 by phosphorylation at threonine 508 within the activation loop. J Biol Chem 275:3577-3582.

Patel RA, Forinash KD, Pireddu R, Sun Y, Sun N, Martin MP, Schönbrunn E, Lawrence NJ, and Sebti SM (2012) RKI-1447 is a potent inhibitor of the Rhoassociated ROCK kinases with anti-invasive and antitumor activities in breast cancer. Cancer Res 72:5025-5034.

Rasmussen KD, Simmini S, Abreu-Goodger C, Bartonicek N, Di Giacomo M, Bilbao-Cortes D, Horos R, Von Lindern M, Enright AJ, and O'Carroll D (2010) The miR-144/451 locus is required for erythroid homeostasis. J Exp Med 207: 1351-1358.

Son JW, Kim YJ, Cho HM, Lee SY, Jang JS, Choi JE, Lee JU, Kang MG, Lee YM, Kwon SJ, et al. (2009) MicroRNA expression profiles in Korean non-small cell lung cancer. Tuberc Respir Dis (Seoul) 67:413-421.

Takamura M, Sakamoto M, Genda T, Ichida T, Asakura H, and Hirohashi S (2001) Inhibition of intrahepatic metastasis of human hepatocellular carcinoma by Rho-associated protein kinase inhibitor Y-27632. Hepatology 33: $577-581$

Tili E, Michaille JJ, Costinean S, and Croce CM (2008) MicroRNAs, the immune system and rheumatic disease. Nat Clin Pract Rheumatol 4:534-541.

Tu B, Du L, Fan QM, Tang Z, and Tang TT (2012) STAT3 activation by IL-6 from mesenchymal stem cells promotes the proliferation and metastasis of osteosarcoma. Cancer Lett 325:80-88.
Wang L, Xue L, Yan H, Li J, and Lu Y (2010) Effects of ROCK inhibitor, Y-27632, on adhesion and mobility in esophageal squamous cell cancer cells. Mol Biol Rep 37: 1971-1977.

Wang W, Peng B, Wang D, Ma X, Jiang D, Zhao J, and Yu L (2011) Human tumor microRNA signatures derived from large-scale oligonucleotide microarray datasets. Int $J$ Cancer 129:1624-1634.

Wang W, Zhou X, and Wei M (2015) MicroRNA-144 suppresses osteosarcoma growth and metastasis by targeting ROCK1 and ROCK2. Oncotarget 6:10297-10308.

Wang Y, Zhao W, and Fu Q (2013) miR-335 suppresses migration and invasion by targeting ROCK1 in osteosarcoma cells. Mol Cell Biochem 384:105-111.

Xu D, Takeshita F, Hino Y, Fukunaga S, Kudo Y, Tamaki A, Matsunaga J, Takahash RU, Takata T, Shimamoto A, et al. (2011) miR-22 represses cancer progression by inducing cellular senescence. J Cell Biol 193:409-424.

Zheng B, Liang L, Wang C, Huang S, Cao X, Zha R, Liu L, Jia D, Tian Q, Wu J, et al (2011) MicroRNA-148a suppresses tumor cell invasion and metastasis by downregulating ROCK1 in gastric cancer. Clin Cancer Res 17:7574-7583.

Address correspondence to: Dr. Xiao Ling Zhang, Department of Orthopedic Surgery, Xin Hua Hospital Affiliated to Shanghai Jiao Tong University School of Medicine (SJTUSM), No.1665, Kongjiang Road, Shanghai 200092, People's Republic of China. E-mail: xlzhang@shsmu.edu.cn 